\title{
The comparison of different daidzein-PLGA nanoparticles in increasing its oral bioavailability
}

This article was published in the following Dove Press journal:

International Journal of Nanomedicine

I February 2012

Number of times this article has been viewed

\section{Yiran Ma \\ Xinyi Zhao \\ Jian Li \\ Qi Shen}

School of Pharmacy, Shanghai Jiao Tong University, Shanghai, China
Correspondence: Qi Shen School of Pharmacy, Shanghai Jiao Tong University, Dongchuan Road 800,

Shanghai 200240, China

$\mathrm{Tel}+862$ I 34204049

Fax +86 21 34204049

Email qshen@sjtu.edu.cn
Abstract: The aim of this research was to increase the oral bioavailability of daidzein by the formulations of poly(lactic-co-glycolic) acid (PLGA) nanoparticles loaded with daidzein. Amongst the various traditional and novel techniques of preparing daidzein-loaded PLGA nanoparticles, daidzein-loaded phospholipid complexes PLGA nanoparticles and daidzein-loaded cyclodextrin inclusion complexes PLGA nanoparticles were selected. The average drug entrapment efficiency, particle size, and zeta potential of daidzein-loaded phospholipid complexes PLGA nanoparticles and daidzein-loaded cyclodextrin inclusion complexes PLGA nanoparticles were $81.9 \% \pm 5 \%$, $309.2 \pm 14.0 \mathrm{~nm},-32.14 \pm 2.53 \mathrm{mV}$ and $83.2 \% \pm 7.2 \%, 323.2 \pm 4.8 \mathrm{~nm},-18.73 \pm 1.68 \mathrm{mV}$, respectively. The morphological characterization of nanoparticles was observed with scanning electron microscopy by stereological method and the physicochemical state of nanoparticles was valued by differential scanning calorimetry. The in vitro drug-release profile of both nanoparticle formulations fitted the Weibull dynamic equation. Pharmacokinetic studies demonstrated that after oral administration of daidzein-loaded phospholipid complexes PLGA nanoparticles and daidzein-loaded cyclodextrin inclusion complexes PLGA nanoparticles to rats a dose of $10 \mathrm{mg} / \mathrm{kg}$, relative bioavailability was enhanced about 5.57- and 8.85-fold, respectively, compared to daidzein suspension as control. These results describe an effective strategy for oral delivery of daidzein-loaded PLGA nanoparticles and might provide a fresh approach to enhancing the bioavailability of drugs with poor lipophilic and poor hydrophilic properties.

Keywords: daidzein, phospholipid complexes, cyclodextrin inclusion complexes, PLGA, nanoparticles

\section{Introduction}

Daidzein (4',7-dihydroxylisoflavone) is a water-insoluble isoflavone, ${ }^{1}$ isolated mainly from leguminous plants, which is used in the treatment of hypertension, coronary heart disease, cerebral thrombosis, and menopause syndrome. Recently, studies demonstrated that daidzein could inhibit the growth of cancer cells by activating a cell death pathway and prevent the onset of diabetes. ${ }^{2-4}$ Thus, daidzein is a promising multipurpose treatment. However, the oral bioavailability of daidzein is very poor, which limits its curative effect. ${ }^{5-8}$ Animal experiments also showed that the absolute bioavailability of daidzein suspension after oral administration to rats was only $6.1 \%{ }^{6}$ There is a consensus that the low bioavailability of daidzein is related to the drug's physicochemical properties which include low solubility, low partition coefficient of oil/water, and, in particular, strong metabolism that occurs in the intestine and liver. ${ }^{9}$ Many microparticulate systems have been used in an attempt to address this issue, such as self-microemulsion and solid lipid nanoparticles drug delivery systems, and 
have demonstrated that bioavailability of daidzein increased by 2.5-, 2.9-, and 6.9-fold. ${ }^{10-13}$ An oral route of administration - attractive due to its convenience, patient acceptance, and cost-effectiveness, particularly in the case of chronic therapies - was developed. ${ }^{14}$ In recent years, nano-based drug delivery systems, such as polymeric nanoparticles, have demonstrated obvious advantages in improving the oral bioavailability and pharmacological activities of poorly soluble drugs; ${ }^{15}$ in particular, nanoparticles of synthetic polyester such as poly(lactic-co-glycolic) acid (PLGA) is often chosen due to its biocompatibility and versatility in encapsulating a variety of drugs. ${ }^{16-18}$ Oral delivery of PLGA nanoparticles has also been well studied. ${ }^{19}$ Some of the commonly reported methods of preparing PLGA nanoparticles include emulsification-solvent evaporation and double-emulsification, which represent easy and reproducible techniques that have been widely used by several research groups to prepare nanoparticles. ${ }^{20}$ However, the incorporation of daidzein into polymeric-based nanocarriers may be greatly restricted by these methods due to poor lipophilicity and poor hydrophilicity. To overcome these kinds of issues, efforts have been made to increase affinity for hydrophobic carrier materials of poor lipophilicity drugs. ${ }^{11,21}$ Additionally, cyclodextrins, well-known cyclic oligosaccharides with a lipophilic central cavity and a hydrophilic outer surface, are able to form inclusion complexes with insoluble molecules like camptothecin. ${ }^{22}$ Therefore, by improving the liposolubility and solubility of daidzein with these techniques combined with the benefits of PLGA nanoparticles, it may be feasible to increase the oral bioavailability of daidzein.

Based on previous information, the aim of this work was, firstly, to separately prepare daidzein phospholipid complexes and daidzein cyclodextrin inclusion complexes and encapsulate into PLGA nanoparticles. Secondly, the physicochemical characteristics of nanoparticles were evaluated. Scanning electron microscopy (SEM) was used to characterize particle morphology, while the physical status of daidzein inside the nanoparticles was investigated by differential scanning calorimetry. Finally, an in vitro release test was used to analyze drug release behavior, and blood concentration was measured after oral administration to rats to measure the bioavailability of daidzein. This study concluded that PLGA combined with phospholipid or cyclodextrin as a carrier material could be applied to water soluble and poorly water soluble compounds, respectively, to control drug release. This is the first report which couples these two formulations with daidzein to improve its oral bioavailability and suggests that this innovative strategy could be effective in improving the oral absorption of poor hydrophilic and poor lipophilic drugs.

\section{Materials}

Daidzein (purity $>98 \%$ ) was obtained from Qingze Medical Technology Development Co Ltd (Nanjing, China). PLGA (lactic:glycolic 50:50, molecular weight [Mw] 15,000 Da), phosphatidylcholine, hydroxypropyl-beta-cyclodextrin (Mw $1400 \mathrm{Da}$ ), acetonitrile, methylene chloride, chloroform, and dimethyl sulfoxide were purchased from Sinopharm Chemical Reagent Co Ltd (Shanghai, China). Polyvinyl alcohol (PVA, viscosity 11-14 cp) was obtained from Kayon Biological Technology Co Ltd (Shanghai, China). Distilled water was produced by a Milli-Q ${ }^{\circledR}$ purification system (Millipore Corporation, Billerica, MA). The rest of the chemicals and reagents used were of analytical grade.

\section{Methods \\ Solubility studies}

Before the preparation of daidzein-loaded nanoparticles, the solubilization process of daidzein was studied. Briefly, an excess amount of daidzein was added into $1 \mathrm{~mL}$ of water, methylene chloride, chloroform, or 2\% PVA aqueous solution in a centrifugal tube. Daidzein-phospholipid complexes were added into $1 \mathrm{~mL}$ of methylene chloride or chloroform and a certain amount of daidzein-cyclodextrin inclusion complexes were added into $1 \mathrm{~mL}$ of water or $2 \%$ PVA aqueous solution. These samples were shaken reciprocally at $25^{\circ} \mathrm{C}$ for 48 hours. This preexperiment showed that daidzein could reach solubility equilibrium and keep stability in 48 hours. After this period of time, the suspensions were filtered through a $0.45 \mu \mathrm{m}$ membrane filter (Millipore) in order to remove the undissolved daidzein. The concentration of daidzein in the filtrate was measured by high performance liquid chromatography (HPLC) after appropriate dilution with methanol. The solubility experiments were conducted in triplicate and no interferences in the analysis of daidzein from the presence of both phosphatidylcholine and hydroxypropyl-betacyclodextrin were found.

\section{Preparation of daidzein complexes Daidzein-phospholipid complexes preparation}

A schematic diagram of the formation of daidzein-loaded phospholipid complexes PLGA nanoparticles (D-PNPs) and daidzein-loaded cyclodextrin inclusion complexes PLGA nanoparticles (D-CNPs) is shown in Figure 1. As described in Figure 1A, daidzein-phospholipid complexes were prepared according to a previous report in which insulin-phospholipid 


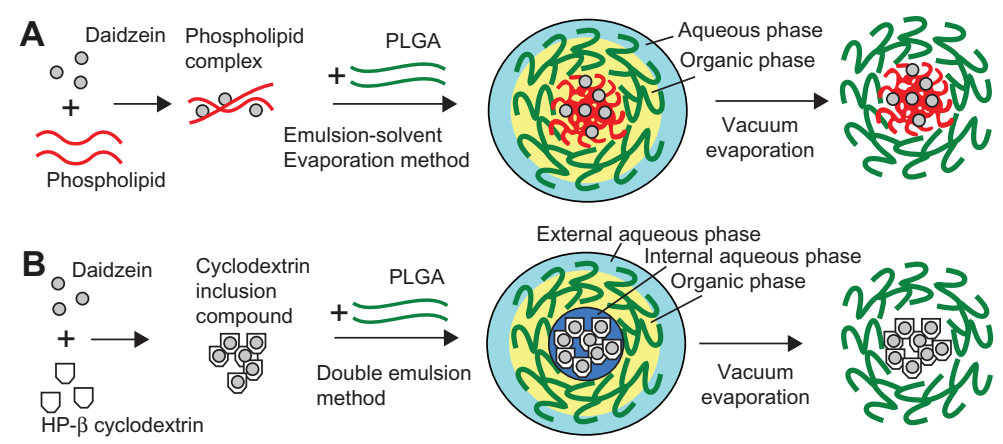

Figure I Schematic diagram of the formations of daidzein-loaded phospholipid complexes poly(lactic-co-glycolic) acid nanoparticles and daidzein-loaded cyclodextrin inclusion complexes poly(lactic-co-glycolic) acid nanoparticles.

Abbreviations: HP- $\beta$ cyclodextrin, hydroxypropyl-beta-cyclodextrin; PLGA, poly(lactic-co-glycolic) acid.

complexes were constructed. ${ }^{23}$ In brief, daidzein ( $2 \mathrm{mg}$ ) was added to $5 \mathrm{~mL}$ ethanol, containing $20 \mathrm{mg}$ phosphatidylcholine, in a water bath at $40^{\circ} \mathrm{C}$. After stirring for 12 hours, ethanol was removed by nitrogen gas and the residues were collected to obtain the daidzein-phospholipid complexes.

\section{Daidzein-cyclodextrin inclusion complexes preparation}

Daidzein-cyclodextrin inclusion complexes were prepared by a fluid inclusion method, ${ }^{22}$ which is shown in Figure 1B. Briefly, daidzein was added to $5 \mathrm{~mL}$ methanol, containing 1:10 molar ratio of hydroxypropyl-beta-cyclodextrin, at $30^{\circ} \mathrm{C}$. After stirring for 12 hours, methanol was volatilized by nitrogen gas and the residues were collected.

\section{Preparation of nanoparticles D-PNPs preparation}

As shown in Figure 1A, D-PNPs were prepared by an emulsion-solvent evaporation method. ${ }^{23}$ Briefly, the obtained daidzein-phospholipid complexes were dissolved in $2 \mathrm{~mL}$ of methylene chloride containing $60 \mathrm{mg}$ PLGA previously dissolved. The organic phase was emulsified in $20 \mathrm{~mL}$ of aqueous phase containing PVA (1\%, weight/volume [w/v]) by sonication over an ice-bath using a Scientz-IID ultrasonic probe (LifeScientz Bio-technology Co, Ltd, Ningbo, China) at an output of $450 \mathrm{~W}$ for 5 minutes to form an oil-in-water $(\mathrm{o} / \mathrm{w})$ emulsion. Then, the organic solvent was evaporated under vacuum at a temperature of $30^{\circ} \mathrm{C}$ in a rotary evaporator (RE52CS; Xian Depai Biotechnology Co, Ltd, Shaanxi, China). The residues were solidified by the addition of sodium chloride aqueous solution $(1 \%, \mathrm{w} / \mathrm{v})$ at $0^{\circ} \mathrm{C}$. After the nanosuspensions were centrifuged and washed three times with ultra-pure water, the formulations were frozen and freeze-dried (ALPHA 1-2/ LDplus; Martin Christ GmbH, Osterode am Harz, Germany) using trehalose ( $2 \%$, weight/weight) as a cryoprotectant.

\section{D-CNPs preparation}

As shown in Figure 1B, D-CNPs were prepared by a double emulsion method..$^{20}$ Briefly, $1.0 \mathrm{~mL}$ of PVA aqueous solution $(2 \%, w / v)$ containing daidzein-cyclodextrin inclusion complexes was emulsified in $2 \mathrm{~mL}$ of chloroform, containing $20 \mathrm{mg}$ phosphatidylcholine and $60 \mathrm{mg}$ PLGA, by high-speed homogenization at $10,000 \mathrm{rpm}$ for 2 minutes. The primary emulsion was added to $20 \mathrm{~mL}$ of PVA solution $(1 \%$, w/v) and sonicated at $450 \mathrm{~W}$ for 5 minutes to form a double-emulsion. Organic solvent residues were removed and the following procedures were the same as described above.

\section{HPLC analytical method}

The amount of daidzein loaded into nanoparticles was evaluated by HPLC. Briefly, the chromatography system used was composed of a Shimadzu LC-20AT chromatographic system with an LC-20AT binary pump and an SPD-20A ultraviolet-visible detector (Shimadzu Corporation, Tokyo, Japan). Data processing was performed with LCsolution software (v1.0.0.1; Shimadzu). Analysis was carried out on a Dikma Dimonsil $\mathrm{C}_{18}$ column $(200 \mathrm{~mm} \times 4.6 \mathrm{~mm}, 5 \mu \mathrm{m}$; Dikma Technologies, Inc, Beijing China). The mobile phase was composed of methanol and deionized water (55:45 volume/volume), ${ }^{9}$ and the flow rate was $1.0 \mathrm{~mL} /$ minute. Column temperature was maintained at $25^{\circ} \mathrm{C}$, ultravioletvisible detection wavelength was set at $249 \mathrm{~nm}$, and injection volume was $20 \mu \mathrm{L}$.

\section{Characterization of nanoparticles}

Particle size and zeta potential of nanoparticles were determined by a particle size analyzer (90Plus; Brookhaven Instruments Corporation, Holtsville, NY). Nanoparticle suspension was analyzed by autocorrelation to determine both mean size and zeta potential. All measurements were performed in triplicate. 
Morphological evaluation of the nanoparticles was performed using SEM (FEI ${ }^{\text {TM }}$ Sirion 200/Inca Oxford; FEI Company, Hillsboro, OR). Briefly, a drop of the dilute nanoparticle suspension was placed onto a copper sheet and then dried under reduced pressure at $40^{\circ} \mathrm{C}$. For SEM analysis, the surfaces of the corresponding membranes were covered with gold in a vacuum before viewing under the microscope.

Thermogram characteristics of selected batches of nanoparticles were determined by differential scanning calorimetry (Pyris 1; PerkinElmer, Waltham, MA). Samples (blank-PNPs, blank-CNPs, D-PNPs, D-CNPs, and pure daidzein as a control - calculated by drug loading which contained $2 \mathrm{mg}$ daidzein) were placed separately in a series of sealed aluminum pans and detected. Samples were scanned at $200^{\circ} \mathrm{C}-400^{\circ} \mathrm{C}$ and heated at a rate of $10^{\circ} \mathrm{C} /$ minute. $^{24}$

Drug encapsulation efficiency and drug loading were analyzed by HPLC method. Briefly, $100 \mu \mathrm{L}$ of evaporated nanoparticle suspension was sucked out and mixed with $900 \mu \mathrm{L}$ dimethyl sulfoxide to destruct the nanoparticles. After being vortexed for 30 seconds, $100 \mu \mathrm{L}$ of sample was withdrawn and $900 \mu \mathrm{L}$ of methanol was added to precipitate insoluble polymers. After the mixture was vortexed again and centrifuged, the supernatant was filtered using a $0.45 \mu \mathrm{m}$ membrane filter (Millipore) and the result was calculated as $\mathrm{W}_{\text {total }}$. Similarly, the amount of daidzein entrapped in the nanoparticles was determined by the following procedure: evaporated nanoparticle suspension $(100 \mu \mathrm{L})$ was sucked out and centrifuged $(12,000 \mathrm{rpm}$, 30 minutes) in order to remove the nonentrapped daidzein. Then, $1 \mathrm{~mL}$ dimethyl sulfoxide was added to destruct the nanoparticles. After being vortexed for 30 seconds, $100 \mu \mathrm{L}$ of sample was withdrawn and $900 \mu \mathrm{L}$ of methanol was added to precipitate insoluble polymers. After the mixture was vortexed again and centrifuged, the supernatant was filtered using a $0.45 \mu \mathrm{m}$ membrane filter (Millipore) and the yield was calculated as $\mathrm{W}_{\text {entrapped }}$. Triplicate samples were prepared for each of the formulations. Drug encapsulation efficiency (\%) and drug loading (\%) were calculated by the following equations:

$$
\begin{gathered}
\text { Encapsulation efficiency }=\left(\mathrm{W}_{\text {entrapped }} / \mathrm{W}_{\text {total }}\right) \times 100 \\
\text { Drug loading }=\left(\mathrm{W}_{\text {entrapped }} / \mathrm{W}_{\mathrm{NPs}}\right) \times 100
\end{gathered}
$$

where $\mathrm{W}_{\text {total }}$ was the total amount of daidzein, $\mathrm{W}_{\text {entrapped }}$ was the amount of encapsulated drug, and $\mathrm{W}_{\mathrm{NPs}}$ was the weight of the freeze-dried nanoparticles.

\section{In vitro release study}

The in vitro release study was carried out using the dialysis bag diffusion technique. The release rate of daidzein from the nanoparticles was measured by HPLC and performed in a thermo shaker bath system (HZ-9212S Shaking Incubator; Taicang Science \& Technology Equipment Inc, Taicang, China). which was maintained at $37^{\circ} \mathrm{C}$ and shaken horizontally at $100 \mathrm{rpm}$. Freeze-dried nanoparticles (calculated by drug loading which contained $1 \mathrm{mg}$ daidzein and determined at the first time interval in the dissolution media by HPLC) or $1 \mathrm{mg}$ pure daidzein was placed in a dialysis membrane bag with a molecular cut-off of $3500 \mathrm{Da}$ (Shanghai GreenBird Science \& Technology Development Co Ltd, Shanghai, China), all groups were in sink condition with this amount of drug. Then, each group of samples was immersed in $30 \mathrm{~mL}$ of phosphate buffer solution ( $\mathrm{pH} 7.4)$. After a particular time interval $(0,1,2,4,6,12,24,36,48,60$, and 72 hours), $1 \mathrm{~mL}$ of the dissolution medium was withdrawn and the nanoparticles were resuspended in medium which was replaced with equal volume of fresh dissolution medium at the same temperature. The released daidzein in the dissolution media was determined by HPLC. Measurements were performed in triplicate for each batch.

\section{Bioavailability studies}

In vivo pharmacokinetics study was carried out using male Sprague Dawley rats $(250 \pm 10 \mathrm{~g})$. The animal experimental protocols were performed according to the guidelines of the Experimental Animal Ethics Committee of Shanghai Jiao Tong University (Shanghai, China). These healthy rats were randomly divided into three treatment groups and fasted overnight with free access to water before drug administration. They were administered orally with D-PNPs, D-CNPs, or daidzein suspension (daidzein dispersed in $0.5 \%$ sodium carboxymethyl cellulose solution) at a dose of $10 \mathrm{mg} / \mathrm{kg}$. After administration, $300 \mu \mathrm{L}$ blood samples were collected from the retroorbital plexus at predetermined time points of $0.5,1,1.5$, $2,4,8,12,24,36$, and 48 hours and placed into heparinized microcentrifuge tubes (100 IU/mL blood). The blood samples were centrifuged at $5000 \mathrm{rpm}$ for 5 minutes and the separated plasma samples were stored at $-80^{\circ} \mathrm{C}$ until analysis.

Daidzein concentration in plasma, with $0.5 \mu \mathrm{g} / \mathrm{mL}$ ethylparaben as the internal standard, was determined by HPLC method. Plasma samples were prepared in the following steps: $100 \mu \mathrm{L}$ plasma was firstly mixed with $10 \mu \mathrm{L}$ internal standard and then $900 \mu \mathrm{L}$ acetonitrile was added to the plasma. Vortexed for 5 minutes, the plasma sample was centrifuged at $12,000 \mathrm{rpm}$ for 10 minutes. After centrifuging, 
the supernatant was taken out, dried by nitrogen gas, and the residue was reconstituted in $100 \mu \mathrm{L}$ methanol. Following further centrifugation at $12,000 \mathrm{rpm}$ for 10 minutes, $20 \mu \mathrm{L}$ of sample solution was injected into the HPLC system. Peak concentration and time to reach peak concentration were determined directly from the plasma concentrationtime curves. Area under the curve was calculated by the trapezoidal method from zero to the final sampling time.

\section{Statistics}

All experiments in this study were performed at least three times and results expressed as mean \pm standard deviation. Statistical significance was assessed by Student's $t$-test or Dunnett's test for multiple comparisons with $P<0.05$ as the minimal level of significance. Pharmacokinetic analysis was performed using Kinetica software program (v4.4; Thermo Fisher Scientific, Waltham, MA).

\section{Results and discussion Solubility study}

In order to study the role of complexes in promoting the solubility of daidzein, a solubility experiment was designed. As shown in Table 1, daidzein is not only poorly soluble in water $(3.84 \mu \mathrm{g} / \mathrm{mL})$ and $2 \%$ PVA aqueous solution $(9.75 \mu \mathrm{g} / \mathrm{mL})$, but also insoluble in the majority of organic solvents that are commonly used in preparation of nanoparticles, eg, methylene chloride $(1.49 \mu \mathrm{g} / \mathrm{mL})$ and chloroform $(0.44 \mu \mathrm{g} / \mathrm{mL})$. Interestingly, solubility of daidzein-phospholipid complexes in dichloromethane increased significantly, ie, 96 times $(828.74 \mu \mathrm{g} / \mathrm{mL})$ higher than its prototype. Similarly, solubility of daidzein-cyclodextrin inclusion complexes increased 95-fold to $757.92 \mu \mathrm{g} / \mathrm{mL}$ in $2 \%$ PVA compared to its prototype.

Technically, for a drug to be encapsulated in PLGA nanoparticles, it must be either lipophilic and combine with PLGA molecular chains by a single o/w method or hydrophilic and be encapsulated in an internal aqueous phase of nanoparticles by a double water-in-oil-in-water (w/o/w) emulsification method. Therefore, this must be the main factor limiting daidzein formulation development, which strongly suggests that it is possible to encapsulate daidzein in PLGA nanoparticles if the solubility is changed.

In the present study, to increase lipid or aqueous solubility of daidzein, two different kinds of complexes using phospholipid or cyclodextrin were prepared. Then, nanoparticle formulations of these complexes were prepared by different methods due to their different solubility characteristics. Daidzeinphospholipid complexes as lipophilic substances were easily encapsulated into PLGA nanoparticles by a single o/w method and daidzein-cyclodextrin inclusion complexes as hydrophilic substances were encapsulated in an internal aqueous phase of PLGA nanoparticles by a double w/o/w method.

\section{Optimization of the preparation method}

As shown in Figure 1, the most important step was modifying the drug before it was encapsulated into the nanoparticles. In the schematic diagram of D-PNPs (Figure 1A), daidzein was first combined with phospholipid to form daidzein-phospholipid complexes and then totally codissolved in dichloromethane with PLGA. In the next ultrasound step, the daidzein-phospholipid complexes were fixed inside the PLGA lipophilic chain side while the oil phase formed droplets in water. This was the key step in the preparation of D-PNPs formation. Dichloromethane evaporation maintained the shape of the D-PNPs and created particles that could not fuse to one another.

Compared to D-PNPs, the schematic for preparation of D-CNPs (Figure 1B) is more complicated. After the preparation of the daidzein-cyclodextrin inclusion complexes, a single emulsion was formed by high-speed mixing of the internal aqueous phase containing daidzein-cyclodextrin inclusion complexes and the organic phase containing PLGA and phosphatidylcholine. A stable w/o single emulsion was the key step in forming D-CNPs as the structure kept daidzein inside the PLGA layer. A w/o/w double emulsion was prepared after the single emulsion was obtained, and chloroform was evaporated to yield D-CNPs.

As a safe drug delivery system, PLGA nanoparticles have been used for hundreds of drugs in modern pharmaceutical research, but never for daidzein. Many traditional

Table I Solubility of pure daidzein and daidzein in each complex form in different solvents

\begin{tabular}{llll}
\hline Solvent & Daidzein $(\mu \mathrm{g} / \mathrm{mL})$ & $\begin{array}{l}\text { Daidzein-phospholipid } \\
\text { complex }(\mu \mathrm{g} / \mathrm{mL})\end{array}$ & $\begin{array}{l}\text { Daidzein-cyclodextrin inclusion } \\
\text { complex }(\mu \mathrm{g} / \mathbf{m L})\end{array}$ \\
\hline $\mathrm{H}_{2} \mathrm{O}$ & $3.84 \pm 0.13$ & - & $734.65 \pm 15.9 \mathrm{I}$ \\
$2 \% \mathrm{PVA}$ & $9.75 \pm 0.37$ & - & $757.92 \pm 18.78$ \\
$\mathrm{CH}_{2} \mathrm{Cl}_{2}$ & $1.49 \pm 0.08$ & $828.74 \pm 16.13$ & - \\
$\mathrm{CHCl}_{3}$ & $0.44 \pm 0.03$ & $697.30 \pm 13.47$ & - \\
\hline
\end{tabular}

Note: Mean \pm standard deviation, $\mathrm{n}=3$.

Abbreviations: $\mathrm{CH}_{2} \mathrm{Cl}_{2}$, dichloromethane; $\mathrm{CHCl}_{3}$, chloroform; $\mathrm{H}_{2} \mathrm{O}$, water; PVA, polyvinyl alcohol. 
nanotechnical methods have been used previously in the authors' lab for encapsulating daidzein directly into PLGA nanoparticles, including the precipitation method, ${ }^{25,26}$ thin-film rehydration method, ${ }^{27,28}$ and emulsification-solvent evaporation method..$^{29,30}$ The nanoprecipitation technique for preparation of nanoparticles is based on the interfacial deposition of a polymer following displacement of a semipolar solvent miscible with water from a lipophilic solution. However, this method suffers the drawback of poor incorporation efficiency of poor lipophilic drugs due to rapid migration and, therefore, loss of drug into the aqueous phase. Compared to the nanoprecipitation method, thin film hydration technique for preparation of nanoparticles is suitable for lipid-soluble drugs, which poses a significant challenge to encapsulating poor lipophilic drugs directly into water-insoluble polymers efficiently. The emulsification-solvent evaporation method also is another commonly used method for preparation of nanoparticles. ${ }^{30}$ However, one of the disadvantages of the traditional emulsification-solvent evaporation method is low encapsulation efficiencies of poor lipophilic drugs (for o/w emulsification method) or low encapsulation efficiencies of poor hydrophilic drugs (for w/o/w emulsification method). For o/w emulsification method, the drug would diffuse out or partition from the dispersed oil phase into the aqueous continuous phase and microcrystalline fragments of the hydrophilic drugs get deposited on the nanoparticle surface. This would result in low trapping of poor lipophilic drugs. The o/w emulsification process is therefore widely used to encapsulate lipid-soluble drugs. For w/o/w emulsification method, poor hydrophilic drugs would diffuse out or partition from the dispersed internal aqueous phase into the oil phase and this would result in low trapping of poor hydrophilic drugs. These traditional methods were compared to the ones created in this study, and the results are shown in Figure 2. Unfortunately, the results show that encapsulation efficiency and drug loading of nanoparticles obtained from traditional methods were very low (encapsulation efficiency $<25 \%$ and drug loading $<0.5 \%$ ). Surprisingly, both D-PNPs and D-CNPs exhibited better encapsulation efficiency and drug loading (encapsulation efficiency $>80 \%$ and drug loading $>1 \%$ for both; D-CNPs drug loading was much higher than D-PNPs).

These results confirm the assumption that increased affinity of daidzein to the organic phase or aqueous phase increases the amount of drug in PLGA or reduces the loss of drug in the external aqueous phase during the emulsification step. In the traditional methods mentioned above, the majority of daidzein cannot encapsulate into PLGA nanoparticles directly because daidzein cannot coexist in the organic phase (dichloromethane) with PLGA or in the internal aqueous phase with $2 \%$ PVA. Thus, daidzein did not appear to be suitable for encapsulation in PLGA nanoparticles directly using traditional methods. After formation of phospholipid complexes or cyclodextrin inclusion complexes, daidzein accumulated in either the organic phase or internal aqueous phase to substantially improve drug loading and encapsulation efficiency.

Overall, daidzein PLGA nanoparticles made by any other method were inferior to D-PNPs and D-CNPs described here (D-PNPs appear inferior to D-CNPs), and these improvements are due to the increased solubility of daidzein following complex formations.

\section{Characterization of D-PNPs and D-CNPs}

Preliminary studies were undertaken to determine the range of conditions (particle size and encapsulation efficiency) suitable for the formation of nanoparticles. As a first step, the present work more intensively investigated the possibility of forming nanoparticles with different $\mathrm{Mw}$ and concentration of PLGA in the organic phase. PLGA Mw and concentration had a great impact on size and encapsulation efficiency

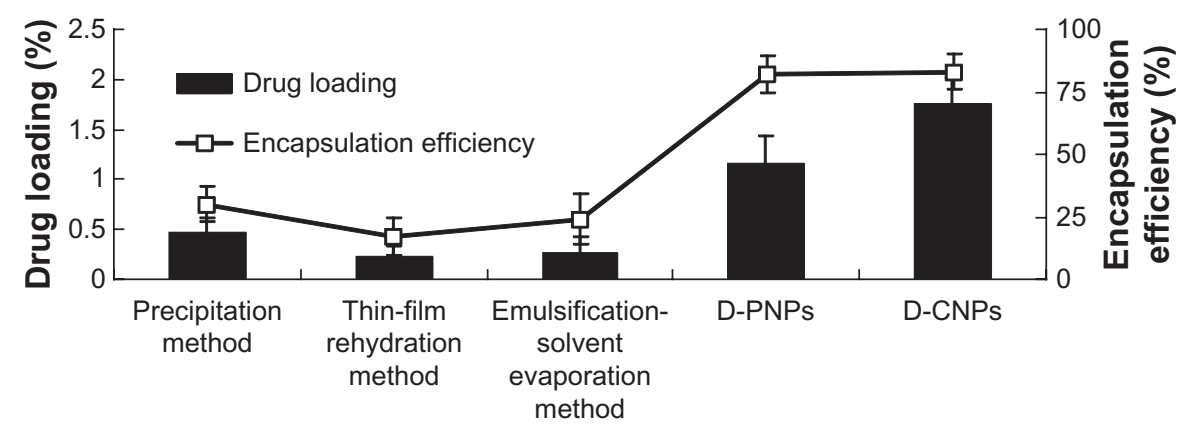

Figure 2 Drug loading and encapsulation efficiency of daidzein-loaded poly(lactic-co-glycolic) acid nanoparticles obtained from traditional methods versus daidzein-loaded phospholipid complexes poly(lactic-co-glycolic) acid nanoparticles and daidzein-loaded cyclodextrin inclusion complexes poly(lactic-co-glycolic) acid nanoparticles. Abbreviations: D-PNPs, daidzein-loaded phospholipid complexes poly(lactic-co-glycolic) acid nanoparticles; D-CNPs, daidzein-loaded cyclodextrin inclusion complexes poly(lactic-co-glycolic) acid nanoparticles. 
of nanoparticles. When PLGA Mw increased from $5000 \mathrm{Da}$ to $15,000 \mathrm{Da}$, size and encapsulation efficiency of particles both increased significantly (data not shown). Similarly, the size and encapsulation efficiency of nanoparticles increased as the concentration of PLGA increased (from $20 \mathrm{mg} / \mathrm{mL}$ to $40 \mathrm{mg} / \mathrm{mL}$ ). When concentration of PLGA in the organic phase increased from $30 \mathrm{mg} / \mathrm{mL}$ to $40 \mathrm{mg} / \mathrm{mL}$, the size of nanoparticles increased significantly and there were no significant differences in encapsulation efficiency (data not shown). This finding is in agreement with other published studies. ${ }^{31,32}$ Taking particle size and drug encapsulation both as evaluation standards, PLGA with Mw of 15,000 Da and concentration of $30 \mathrm{mg} / \mathrm{mL}$ were selected.

Another aspect worth noting is the function of PVA during the preparation process. It was found that nanoparticles containing PVA exhibited higher encapsulation efficiency and small size compared to others. Some studies have also indicated that it was used as an emulsifier in the preparation of nanoparticles. ${ }^{33}$ The present research proposes PVA at $1 \%(\mathrm{w} / \mathrm{v})$ in the aqueous phase as an emulsifier during the preparation process of D-PNPs and D-CNPs, as it was easier to collect nanoparticles at this concentration than at $2 \%(\mathrm{w} / \mathrm{v})$. The reason for PVA's superior performance may be that it was easier to form a thin film on the particle surfaces, which could control particle size and prevent daidzein leakage. In addition, as described in Figure 1, the stability of w/o single emulsion stability is a prerequisite of successful nanoparticle preparation. It was found that dissolved daidzein-cyclodextrin inclusion complexes in the high viscosity of PVA solution at $2 \%$ $(\mathrm{w} / \mathrm{v})$ as the internal aqueous phase increased encapsulation efficiency compared to the group without PVA. Regarding the effect of phosphatidylcholine in the D-CNPs preparation process, it was found that a stable single emulsion could be more easily formed to increase encapsulation efficiency of daidzein in nanoparticles in the presence of an emulsifier (phosphatidylcholine at 10\% of PLGA weight).
After the nanoparticles were isolated, a series of detection experiments were conducted to demonstrate whether the structure and function of nanoparticles were consistent with expectations. Nanoparticle morphology, particle size, surface charge, and physical state of encapsulated drug were studied.

D-PNPs and D-CNPs yielded particles $323.2 \pm 4.8 \mathrm{~nm}$ and $309.2 \pm 14 \mathrm{~nm}$ in size (mean diameter), respectively, and zeta potential of D-PNPs and D-CNPs was $-32.14 \pm 2.53 \mathrm{mV}$ and $-18.73 \pm 1.68 \mathrm{mV}$, respectively. Zeta potential is a function of the surface charge of particle dispersions which is commonly used to predict and control dispersion stability. Higher negative values were obtained for D-PNPs $(-32.14 \pm 2.53 \mathrm{mV})$ and a marked decrease in the surface charge for D-CNPs occurred $(-18.73 \pm 1.68 \mathrm{mV})$. It was speculated that the zeta potential of nanoparticles may be affected by the presence of cyclodextrins, but this theory was not explored in depth.

Encapsulation efficiency of daidzein in D-PNPs was $81.9 \% \pm 5 \%$ and $83.2 \% \pm 7.2 \%$ in D-CNPs. Drug loading of D-PNPs and D-CNPs was $1.27 \% \pm 0.33 \%$ and $1.75 \% \pm 0.24 \%$, respectively, indicating that D-CNPs were more efficient for drug loading than D-PNPs.

The morphological characterization of the optimized D-PNPs and D-CNPs was examined by SEM. SEM is expected to provide information on nanoparticle morphology and size. Examination of SEM photographs of the nanoparticles revealed that the surfaces were smooth and spherical (Figure 3).

The physicochemical state of nanoparticles was valued by differential scanning calorimetry. Figure 4 shows the differential scanning calorimetric thermograms of daidzein, blank-CNPs, blank-PNPs, D-CNPs, and D-PNPs. The melting peak of daidzein appeared at $331^{\circ} \mathrm{C}$ (Figure 4A). The curves of D-CNPs and D-PNPs showed the same endothermic trend as blank-CNPs and blank-PNPs (Figure 4B and C), but did not show the daidzein melting peak (Figure 4D and E).

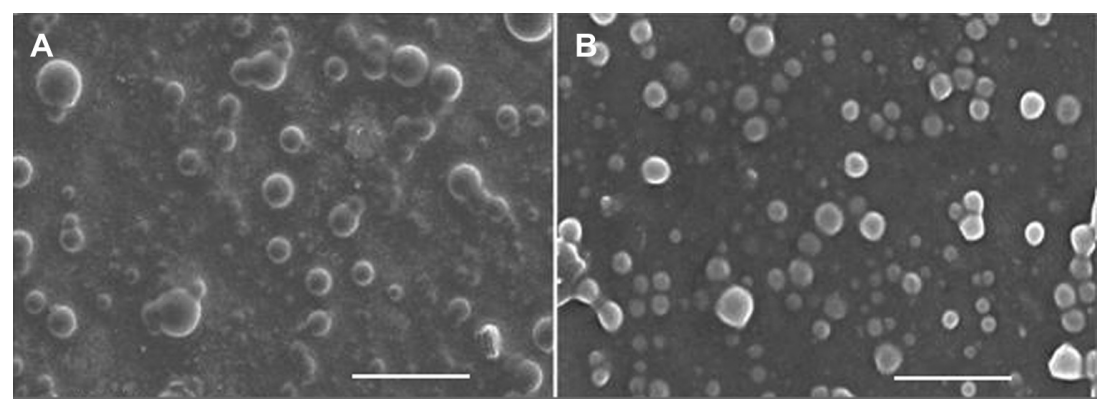

Figure 3 Scanning electron microscopic photographs of daidzein-loaded phospholipid complexes poly(lactic-co-glycolic) acid nanoparticles (A) and daidzein-loaded cyclodextrin inclusion complexes poly(lactic-co-glycolic) acid nanoparticles (B).

Note: Bar represents I $\mu \mathrm{m}(80,000 \times$ magnification). 


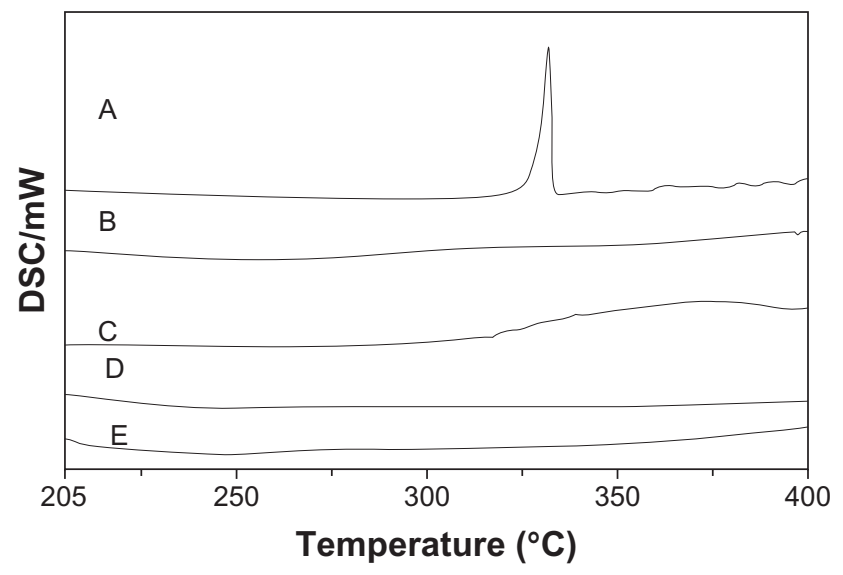

Figure 4 Differential scanning calorimetry curves of daidzein (A), blank phospholipid complexes poly(lactic-co-glycolic) acid nanoparticles (B), blank cyclodextrin inclusion complexes poly(lactic-co-glycolic) acid nanoparticles (C), daidzein-loaded phospholipid complexes poly(lactic-co-glycolic) acid nanoparticles (D), and daidzeinloaded cyclodextrin inclusion complexes poly(lactic-co-glycolic) acid nanoparticles (E). Abbreviation: DSC, differential scanning calorimetry.

This result indicates that daidzein was not in a crystalline state but in an amorphous state in nanoparticles.

Taking into account that the primary goal of this work was to design a nanoparticulate drug delivery system intended for oral administration, a critical study of these particles would be the evaluation of their stability in the different media present in the gastrointestinal tract. PLGA nanoparticle stability in simulated gastrointestinal conditions can be reviewed in the literature. Previous research reported that PLGA nanoparticles were stable in gastric fluid $(\mathrm{pH} 1.2)$ and intestinal fluid (pH 7.5). ${ }^{34,35}$ Therefore, it was speculated that both D-CNPs and D-PNPs were stable in simulated gastrointestinal conditions.

\section{In vitro release}

The in vitro release behavior of the nanoparticles is summarized in the cumulative percentage release shown in Figure 5. The accumulative pure daidzein release rate was only $26.94 \%$ while D-PNPs and D-CNPs achieved $80.85 \%$ and $90.12 \%$, respectively, in 72 hours. It was demonstrated that pure daidzein cannot be released effectively from the dialysis bag, even when the release solution was an efficient sink. This may be because most of the daidzein was attached to the dialysis bag wall or sank to the bag bottom. This explains why the bioavailability of prototype daidzein is low. A possible reason for this was that $1 \mathrm{mg}$ pure daidzein was suspended in $2 \mathrm{~mL}$ of release solution in the dialysis bag, and small clumps of daidzein could have blocked the dialysis bag pores and kept other daidzein molecules from passing through. On the contrary, D-PNPs and D-CNPs were released completely. Each release pattern fitted the Weibull equation:

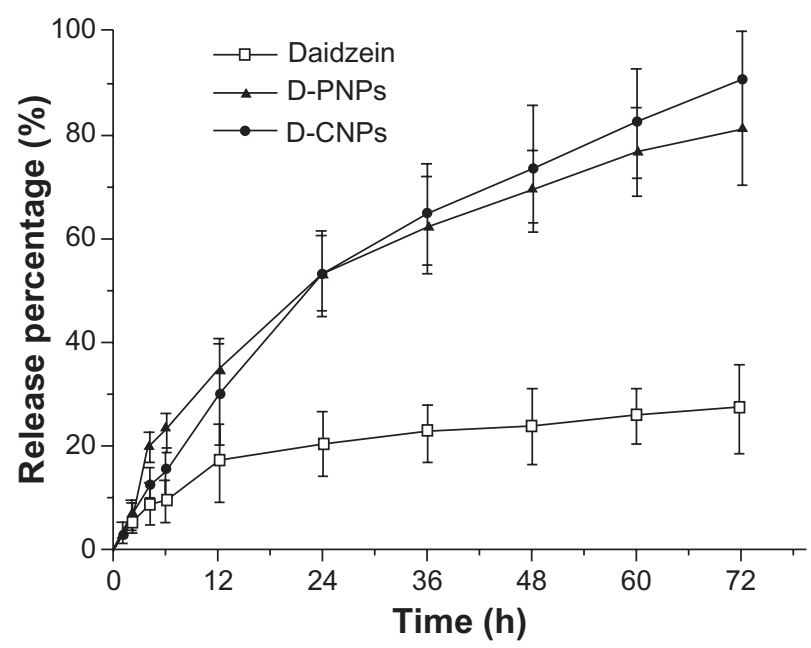

Figure 5 In vitro cumulate release of pure daidzein and daidzein from daidzeinloaded phospholipid complexes poly(lactic-co-glycolic) acid nanoparticles and daidzein-loaded cyclodextrin inclusion complexes poly(lactic-co-glycolic) acid nanoparticles in phosphate buffer solution $\left(\mathrm{pH} \mathrm{7.4)}\right.$ at a temperature of $37^{\circ} \mathrm{C}$ and a rate of $100 \mathrm{rpm}$.

Abbreviations: D-PNPs, daidzein-loaded phospholipid complexes poly(lacticco-glycolic) acid nanoparticles; D-CNPs, daidzein-loaded cyclodextrin inclusion complexes poly(lactic-co-glycolic) acid nanoparticles; h, hours.

$$
\begin{aligned}
& \text { For D-PNPS : } y=1-e^{-0.387 t^{0.928}}\left(\mathrm{R}^{2}=0.998\right) \\
& \text { For D-CNPS }: y=1-e^{-0.33 t^{0.829}}\left(\mathrm{R}^{2}=0.993\right) .
\end{aligned}
$$

There was no significant burst effect in the initial release phase, possibly because the washing process removed all daidzein adhered on the nanoparticle surface. The initial burst release is one of the major problems in the development of controlled release formulations, including drug-loaded microparticles and nanoparticles, especially with low Mw drugs. In the present experiment, both D-PNPs and D-CNPs released stably from the beginning and didn't have a burst release, which is shown by their fit to the Weibull equation and illustrates the advantageous structure of D-PNPs and D-CNPs. In both D-PNPs and D-CNPs, daidzein was encapsulated within PLGA. PVA outside the D-PNPs is an important hydrophilic layer to keep daidzein away from the particle surface, while the three-tier $\mathrm{w} / \mathrm{o} / \mathrm{w}$ double emulsion structure in D-CNPs fixed daidzein in the inner core of the particle.$^{36}$ When nanoparticles were put in a release solution, PVA was dissolved and the carriers (PLGA or phosphatidylcholine) were dissolved gradually to form holes or disintegrated to release the drugs. ${ }^{37}$ This steady release rate from nanoparticles will allow better drug control in vivo.

\section{Bioavailability studies}

D-PNPs, D-CNPs, and daidzein suspension were orally administrated to male Sprague Dawley rats. Plasma concentration-time curves are shown in Figure 6. A remarkable 


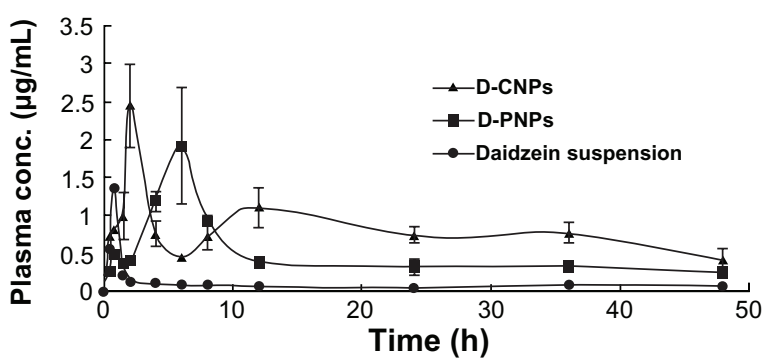

Figure 6 Mean plasma concentration-time profiles of daidzein in rats after oral administration of daidzein-loaded phospholipid complexes poly(lactic-co-glycolic) acid nanoparticles, daidzein-loaded cyclodextrin inclusion complexes poly(lactic-coglycolic) acid nanoparticles, and daidzein suspension at a dose of $10 \mathrm{mg} / \mathrm{kg}(\mathrm{n}=3)$. Abbreviations: conc, concentration; D-PNPs, daidzein-loaded phospholipid complexes poly(lactic-co-glycolic) acid nanoparticles; D-CNPs, daidzein-loaded cyclodextrin inclusion complexes poly(lactic-co-glycolic) acid nanoparticles; h, hours.

increase in plasma concentrations and a much slower release of daidzein were observed when nanoparticle groups were studied compared to control group. This result clearly shows that there was a great difference in bioavailability between the two kinds of nanoparticles and daidzein alone. The profile of the D-CNPs curve can be divided in two parts: in the first part, D-CNPs had a high sharp absorption peak at 2 hours; in the second part D-CNPs had a low absorption peak at 12 hours.

Table 2 shows the pharmacokinetic parameters (peak concentration, time to reach peak concentration, area under the curve ${ }_{0-48}$, half-life, and relative bioavailability) of daidzein after oral administration. As shown in Table 2, peak concentration was found to be significantly higher for D-PNPs $(1.92 \pm 0.69 \mu \mathrm{g} / \mathrm{mL})$ and D-CNPs $(2.44 \pm 0.74 \mu \mathrm{g} / \mathrm{mL})$ than for daidzein suspension group $(1.35 \pm 0.47 \mu \mathrm{g} / \mathrm{mL})$. Similarly, half-life was faster for D-PNPs $(8.62 \pm 2.69$ hours $)$ and D-CNPs $(3.38 \pm 1.24$ hours $)$ compared to daidzein

Table 2 Pharmacokinetic parameters of daidzein after oral administration of daidzein-loaded phospholipid complexes poly(lactic-coglycolic) acid nanoparticles, daidzein-loaded cyclodextrin inclusion complexes poly(lactic-co-glycolic) acid nanoparticles, and daidzein suspension at the dose of $10 \mathrm{mg} / \mathrm{kg}$

\begin{tabular}{llll}
\hline Parameters & $\begin{array}{l}\text { Daidzein } \\
\text { suspension }\end{array}$ & D-PNPs & D-CNPs \\
\hline $\mathrm{C}_{\max }(\mu \mathrm{g} / \mathrm{mL})$ & $\mathrm{I} .35 \pm 0.47$ & $1.92 \pm 0.69$ & $2.44 \pm 0.74^{\mathrm{a}}$ \\
$\mathrm{AUC}_{0-48}(\mathrm{~h} \mu \mathrm{g} / \mathrm{mL})$ & $\mathrm{I} .91 \pm 0.8 \mathrm{I}$ & $10.63 \pm 3.77^{\mathrm{a}}$ & $16.90 \pm 6.93^{\mathrm{b}}$ \\
$\mathrm{T}_{1 / 2}$ (hours) & $\mathrm{I} .15 \pm 0.55$ & $8.62 \pm 2.69^{\mathrm{a}}$ & $3.38 \pm 1.24^{\mathrm{a}}$ \\
$\mathrm{T}_{\max }$ (hours) & $0.76 \pm 0.2 \mathrm{I}$ & $5.97 \pm 2.1^{\mathrm{b}}$ & $2.04 \pm 0.9 \mathrm{I}^{\mathrm{a}}$ \\
$\mathrm{F}_{\text {rel }}(\%)$ & - & 556.98 & 885.62 \\
\hline
\end{tabular}

Notes: ${ }^{a} P<0.05$ compared with daidzein suspension group; ${ }^{b} P<0.01$ compared with daidzein suspension group. Mean \pm standard deviation, $n=3$.

Abbreviations: AUC, area under the curve; $C_{\max }$, peak concentration; D-CNPs, daidzein-loaded cyclodextrin inclusion complexes poly(lactic-co-glycolic) acid nanoparticles; D-PNPs, daidzein-loaded phospholipid complexes poly(lacticco-glycolic) acid nanoparticles; $F_{\text {rel' }}$ relative bioavailability; $T_{\text {max }}$, time to peak concentration; $T_{1 / 2}$, half-life. suspension group ( $1.15 \pm 0.55$ hours $)$ and time to reach peak concentration was also faster for D-PNPs (5.97 \pm 2.17 hours $)$ and D-CNPs (2.04 \pm 0.91 hours) compared to daidzein suspension group $(0.76 \pm 0.21$ hours $)$. In the same way, area under the curve ${ }_{0-48}$ for D-PNPs $(10.63 \pm 3.77 \mathrm{~h} \mu \mathrm{g} / \mathrm{mL})$ and D-CNPs $(16.90 \pm 6.93 \mathrm{~h} \mu \mathrm{g} / \mathrm{mL})$ was five- and eight-fold higher than that of daidzein suspension $(1.91 \pm 0.81 \mathrm{~h} \mu \mathrm{g} / \mathrm{mL})$. Finally, relative bioavailability of D-PNPs and D-CNPs was calculated to be $556.98 \%$ and $885.62 \%$, respectively, compared to daidzein suspension group. These results verify that both D-PNPs and D-CNPs were effective in improving the oral bioavailability of daidzein.

The pharmacokinetic parameters clearly demonstrate that the two kinds of nanoparticles studied greatly improved bioavailability for daidzein. The reasons are many, but among the most salient is the nano-based drug delivery system. On the one hand, particles that are nanoscale in size are easily absorbed into the folds of the intestinal wall, while large particle surface area to volume lends itself to rapid drug dissolution. ${ }^{38-40}$ On the other hand, particulate systems for oral delivery can protect the drug at low $\mathrm{pH}$ levels, facilitate absorption into the intestinal tract, and bypass unwanted metabolic degradation, and the nature of degradation of the carrier maintains a sustained release. ${ }^{41,42}$ Amorphous dispersion of daidzein in nanoparticles might be another reason for better absorption. ${ }^{43,44}$ Apart from these reasons, more importantly, daidzein complexes may play an important role in absorption. Some studies have demonstrated that phospholipid-drug complexes and cyclodextrin-drug complexes could increase oral bioavailability by improving the liposolubility and solubility of drugs. ${ }^{21,22}$ Furthermore, studies have also pointed out that daidzein as a hydrophilic compound could transport across the intestinal barrier via the paracellular pathway and passive transport is the only mechanism of daidzein absorption in the small intestine of rats, at least within the concentrations tested. ${ }^{45}$ In addition, phospholipids have been proven to have a high affinity for flavonoids and accelerates daidzein transport across the cell membrane. ${ }^{46-48}$ Cyclodextrin has been proven as a drug absorption enhancer as well. ${ }^{49,50}$ Since daidzein is a typical poor hydrophilic and poor lipophilic drug with very low oral bioavailability, ${ }^{7}$ the greatly improved liposolubility and solubility of daidzein by D-PNPs and D-CNPs should be favorable to its absorption in the gastrointestinal tract. Daidzein complex intermediated not only the increased drug loading of nanoparticles, but also promoted drug absorption in vivo. 
Somewhat surprisingly, the in vivo bioavailability study of D-PNPs and D-CNPs in the present study showed that the absorption curves were different shapes. D-PNPs had an absorption peak at about 6 hours, while D-CNPs had a high sharp absorption peak at 2 hours and a low absorption peak at 12 hours. It is generally believed that the ileum and colon are the main absorption sites for nanoparticles; as daidzein-phospholipid complexes in D-PNPs are lipophilic substances, and because of their small size, they would be easily taken up by epithelial cells in the ileum and colon as whole nanoparticles or as drug released from PLGA nanoparticles. ${ }^{51,52}$ The absorption process may continue for 8-10 hours with a coincident absorption peak. However, a completely different situation may exist for D-CNPs. As far as is known, daidzein-cyclodextrin inclusion complexes are not likely to be absorbed directly by intestinal epithelial cells, but as hydrophilic compounds they will easily leak out of the nanoparticles into gastric juice. By using cyclodextrin as an absorption enhancer, daidzein would be absorbed easily in the small intestine. The first absorption peak may be due to this process. The second peak that appeared after 10 hours was lower and wider, suggesting that daidzein was absorbed into the blood slowly. Based on previous reports and the concentration-time curve of pure daidzein, the second peak was not caused by entry into hepatic circulation as this did not happen in the prototype daidzein absorption curve. As cyclodextrin cannot pass through intestinal epithelial cells, the most likely explanation was that D-CNPs or daidzein-cyclodextrin inclusion complexes were taken up by lymphocytes in the small intestine and daidzein eventually entered the blood after being transported in microfold cells through the ileum. ${ }^{14,53,54}$

\section{Conclusion}

In the present study, an effective strategy for enhancing the oral absorption of daidzein, which has poor hydrophilicity and poor lipophilicity, was developed. Daidzein was incorporated into PLGA nanoparticles by two improved methods. Both of them used daidzein complexes as intermediates that increased the solubility of daidzein to either aqueous or organic solvents. The soluble complexes were successfully used to form PLGA nanoparticles under optimal preparation conditions. In this experiment, D-PNPs were prepared by an emulsion evaporation method using daidzein-phospholipid complexes as an intermediate, while D-CNPs were prepared by a double emulsion method using daidzein-cyclodextrin inclusion complexes as an intermediate. Detection of nanoparticle properties showed that both structures were consistent with theoretical assumptions. More importantly, the pharmacokinetic results showed that both of them improved oral bioavailability of daidzein in rats. These formulations overcome the problem of poor daidzein encapsulation in the lipophilic polymer carrier PLGA. Two distinct compound-nanoparticles were produced from the same drug by producing and using two different complexes as intermediates. This is a new approach for the formulation of drugs with both poor lipophilic and poor hydrophilic properties.

\section{Acknowledgments}

The work was supported by the National Natural Science Foundation of China (Grant No 30973644), the National Basic Research Program of China (973 Program; Grant No 2007CB936004), and Shanghai Municipal Committee of Science and Technology (Grant No 08DZ1971304).

\section{Disclosure}

The authors report no conflicts of interest in this work.

\section{References}

1. Gutierrez RM, Baez EG. Cardioactive agents from plants. Mini Rev Med Chem. 2009;9(7):878-899.

2. Lo FH, Mak NK, Leung KN. Studies on the anti-tumor activities of the soy isoflavone daidzein on murine neuroblastoma cells. Biomed Pharmacother. 2007;61(9):591-595.

3. Moller FJ, Diel P, Zierau O, HertrampfT, Maass J, Vollmer G. Long-term dietary isoflavone exposure enhances estrogen sensitivity of rat uterine responsiveness mediated through estrogen receptor alpha. Toxicol Lett. 2010;196(3):142-153.

4. Allred CD, Twaddle NC, Allred KF, et al. Soy processing affects metabolism and disposition of dietary isoflavones in ovariectomized BALB/c mice. J Agric Food Chem. 2005;53(22):8542-8550.

5. Janning P, Schuhmacher US, Upmeier A, et al. Toxicokinetics of the phytoestrogen daidzein in female DA/Han rats. Arch Toxicol. 2000;74(8):421-430.

6. Qiu F, Chen XY, Song B, Zhong F, Liu CX. Influence of dosage forms on pharmacokinetics of daidzein and its main metabolite daidzein7-O-glucuronide in rats. Acta Pharmacol Sin. 2005;26(9):1145-1152.

7. Zheng Y, Lee SO, Verbruggen MA, Murphy PA, Hendrich S. The apparent absorptions of isoflavone glucosides and aglucons are similar in women and are increased by rapid gut transit time and low fecal isoflavone degradation. J Nutr. 2004;134(10):2534-2539.

8. Li X, Shen Q, Yuan D, Ma A, Jia W. Determination of daidzein in rat plasma by LC. Chromatographia. 2008;68(3-4):201-205.

9. Kulling SE, Honig DM, Metzler M. Oxidative metabolism of the soy isoflavones daidzein and genistein in humans in vitro and in vivo. J Agric Food Chem. 2001;49(6):3024-3033.

10. Shen Q, Li X, Yuan D, Jia W. Enhanced oral bioavailability of daidzein by self-microemulsifying drug delivery system. Chem Pharm Bull (Tokyo). 2010;58(5):639-643.

11. Zhang Z, Huang Y, Gao F, Bu H, Gua W, LiY. Daidzein-phospholipid complex loaded lipid nanocarriers improved oral absorption: in vitro characteristics and in vivo behavior in rats. Nanoscale. 2011;3(4):1780-1787.

12. Zhang Z, Huang Y, Gao F, et al. A self-assembled nanodelivery system enhances the oral bioavailability of daidzein: in vitro characteristics and in vivo performance. Nanomedicine (Lond). 2011;6(8):1365-1379. 
13. Gao Y, Gu W, Chen L, Xu Z, LiY. The role of daidzein-loaded sterically stabilized solid lipid nanoparticles in therapy for cardio-cerebrovascular diseases. Biomaterials. 2008;29(30):4129-4136.

14. Lavelle EC, Sharif S, Thomas NW, Holland J, Davis SS. The importance of gastrointestinal uptake of particles in the design of oral delivery systems. Adv Drug Deliv Rev. 1995;18(1):5-22.

15. Agüeros M, Zabaleta V, Espuelas S, Campanero MA, Irache JM. Increased oral bioavailability of paclitaxel by its encapsulation through complex formation with cyclodextrins in poly(anhydride) nanoparticles J Control Release. 2010;145(1):2-8.

16. Jain RA. The manufacturing techniques of various drug loaded biodegradable poly(lactide-co-glycolide) (PLGA) devices. Biomaterials. 2000;21(23):2475-2490.

17. Panyam J, Labhasetwar V. Biodegradable nanoparticles for drug and gene delivery to cells and tissue. Adv Drug Deliv Rev. 2003;55(3): 329-347.

18. Shive MS, Anderson JM. Biodegradation and biocompatibility of PLA and PLGA microspheres. Adv Drug Deliv Rev. 1997;28(1): 5-24.

19. Damge C, Aprahamian M, Marchais H, Benoit JP, Pinget M. Intestinal absorption of PLAGA microspheres in the rat. J Anat. 1996;189(Pt 3): 491-501.

20. Tewes F, Munnier E, Antoon B, et al. Comparative study of doxorubicin-loaded poly(lactide-co-glycolide) nanoparticles prepared by single and double emulsion methods. Eur J Pharm Biopharm. 2007;66(3):488-492.

21. Cui F, Shi K, Zhang L, Tao A, Kawashima Y. Biodegradable nanoparticles loaded with insulin-phospholipid complex for oral delivery: preparation, in vitro characterization and in vivo evaluation. J Control Release. 2006;114(2):242-250.

22. Cirpanli Y, Bilensoy E, Lale Dogan A, Çalis S. Comparative evaluation of polymeric and amphiphilic cyclodextrin nanoparticles for effective camptothecin delivery. Eur J Pharm Biopharm. 2009;73(1) 82-89.

23. Jeffery H, Davis SS, O'Hagan DT. The preparation and characterisation of poly(lactide-co-glycolide) microparticles. I: oilin-water emulsion solvent evaporation. Int J Pharm. 1991;77(2-3): $169-175$.

24. Mu L, Feng SS. A novel controlled release formulation for the anticancer drug paclitaxel (Taxol): PLGA nanoparticles containing vitamin E TPGS. J Control Release. 2003;86(1):33-48.

25. Fessi H, Puisieux F, Devissaguet JP, Ammoury N, Benita S. Nanocapsule formation by interfacial polymer deposition following solvent displacement. Int J Pharm. 1989;55:R1-R4

26. Molpeceres J, Guzman M, Aberturas MR, Chacon M, Berges L. Application of central composite designs to the preparation of polycaprolactone nanoparticles by solvent displacement. J Pharm Sci. 1996;85(2):206-213.

27. Aditya NP, Patankar S, Madhusudhan B, Murthy RS, Souto EB. Arthemeter-loaded lipid nanoparticles produced by modified thin-film hydration: pharmacokinetics, toxicological and in vivo anti-malarial activity. Eur J Pharm Sci. 2010;40(5):448-455.

28. Aquilano D, Cavalli R, Gasco MR. Solid lipospheres obtained from hot microemulsions in the presence of different concentrations of cosurfactant the crystallization of stearic acid polymorphs. Thermochim Acta. 1993;230:29-37.

29. Hariharan S, Bhardwaj V, Bala I, Sitterberg J, Bakowasky U, Ravi Kumar MN. Design of estradiol loaded PLGA nanopaticles formulations: a potential oral delivery system for hormone therapy. Pharm Res. 2006;23(1):184-195.

30. Arshady R. Preparation of biodegradable microspheres and microcapsules: 2. Polylactides and related polyesters. J Control Release. 1991;17(1):1-22.

31. Li X, Deng X, Yuan M, et al. Investigation on process parameters involved in preparation of poly-DL-lactide-poly(ethylene glycol) microspheres containing Leptospira Interrogans antigens. Int J Pharm. $1999 ; 178(2): 245-255$
32. Zhu KJ, Jiang HL, Du XY, Wang J, Xu WX, Liu SF. Preparation and characterization of hCG-loaded polylactide or poly(lactideco-glycolide) microspheres using a modified water-in-oil-in-water (w/o/w) emulsion solvent evaporation technique. J Microencapsul. 2001;18(2):247-260.

33. Sahoo SK, Panyam J, Prabha S, Labhasetwar V. Residual polyvinyl alcohol associated with poly(D,L-lactide-co-glycolide) nanoparticles affects their physical properties and cellular uptake. J Control Release. 2002;82(1):105-114.

34. Ahlin P, Kristl J, Kristl A, Vrecer F. Investigation of polymeric nanoparticles as carriers of enalaprilat for oral administration. Int J Pharm. 2002;239(1-2):113-120.

35. Yin Y, Chen D, Qiao M, Wei X, Hu H. Lectin-conjugated PLGA nanoparticles loaded with thymopentin: ex vivo bioadhesion and in vivo biodistribution. J Control Release. 2007;123(1):27-38.

36. Hasan AS, Socha M, Lamprecht A, et al. Effect of the microencapsulation of nanoparticles on the reduction of burst release. Int J Pharm. 2007;344(1-2):53-61.

37. Hurteaux R, Edwards-Levy F, Laurent-Maquin D, Levy MC. Coating alginate microspheres with a serum albumin-alginate membrane: application to the encapsulation of a peptide. Eur J Pharm Sci. 2005;24(2-3):187-197.

38. Ratzinger G, Fillafer C, Kerleta V, Wirth M, Gabor F. The role of surface functionalization in the design of PLGA micro- and nanoparticies. Crit Rev Ther Drug Carrier Syst. 2010;27(1):1-83.

39. Delie F. Evaluation of nano- and microparticle uptake by the gastrointestinal tract. Adv Drug Deliv Rev. 1998;34(2-3):221-233.

40. McClean S, Prosser E, Meehan E, et al. Binding and uptake of biodegradable poly-DL-lactide micro- and nanoparticles in intestinal epithelia. Eur J Pharm Sci. 1998;6(2):153-163.

41. Dong Y, Feng SS. Poly(D,L-lactide-co-glycolide)/montmorillonite nanoparticles for oral delivery of anticancer drugs. Biomaterials. 2005;26(30):6068-6076.

42. O'Hagan DT, Jeffery H, Roberts MJ, McGee JP, Davis SS. Controlled release microparticles for vaccine development. Vaccine. 1991;9(10): $768-771$.

43. Hancock BC, Carlson GT, Ladipo DD, Langdon BA, Mullarney MP. Comparison of the mechanical properties of the crystalline and amorphous forms of a drug substance. Int J Pharm. 2002;241(1): 73-85.

44. Corrigan DO, Healy AM, Corrigan OI. The effect of spray drying solutions of bendroflumethiazide/polyethylene glycol on the physicochemical properties of the resultant materials. Int J Pharm. 2003;262(1-2):125-137.

45. Foti P, Erba D, Spadafranca A, Ciappellano S, Bresciani J, Testolin G. Daidzein is absorbed by passive transport in isolated small intestine of rats. Nutr Res. 2006;26(6):284-288.

46. Fricker G, Kromp T, Wendel A, et al. Phospholipids and lipidbased formulations in oral drug delivery. Pharm Res. 2010;27(8): 1469-1486.

47. Leigh M, van Hoogevest P, Tiemessen H. Optimising the oral bioavailability of the poorly water-soluble drug cyclosporin A using membrane lipid technology. Drug Deliv Syst. 2001;1(3):73-77.

48. Porter CJ, Trevaskis NL, Charman WN. Lipids and lipid-based formulations: optimizing the oral delivery of lipophilic drugs. Nat Rev Drug Discov. 2007;6(3):231-248.

49. Hamada H, Ishihara K, Masuoka N, Mikuni K, Nakajima N. Enhancement of water-solubility and bioactivity of paclitaxel using modified cyclodextrins. J Biosci Bioeng. 2006;102(4):369-371.

50. Mady FM, Abou-Taleb AE, Khaled KA, et al. Evaluation of carboxymethyl-beta-cyclodextrin with acid function: improvement of chemical stability, oral bioavailability and bitter taste of famotidine. Int J Pharm. 2010;397(1-2):1-8.

51. Maiti K, Mukherjee K, Gantait A, Saha BP, Mukherjee PK. Curcumin-phospholipid complex: preparation, therapeutic evaluation and pharmacokinetic study in rats. Int J Pharm. 2007;330(1-2): 155-163. 
52. Sachs-Barrable K, Lee SD, Wasan EK, Thornton SJ, Wasan KM. Enhancing drug absorption using lipids: a case study presenting the development and pharmacological evaluation of a novel lipid-based oral amphotericin B formulation for the treatment of systemic fungal infections. Adv Drug Deliv Rev. 2008;60(6):692-701.

53. Win KY, Feng SS. Effects of particle size and surface coating on cellular uptake of polymeric nanoparticles for oral delivery of anticancer drugs. Biomaterials. 2005;26(15):2713-2722.
54. Florence AT, Hillery AM, Husain N, Jani PU. Nanoparticles as carriers for oral peptide absorption: studies on particle uptake and fate. J Control Release. 1995;36(1-2):39-46.

\section{Publish your work in this journal}

The International Journal of Nanomedicine is an international, peerreviewed journal focusing on the application of nanotechnology in diagnostics, therapeutics, and drug delivery systems throughout the biomedical field. This journal is indexed on PubMed Central, MedLine, CAS, SciSearch ${ }^{\circledR}$, Current Contents ${ }^{\circledR} /$ Clinical Medicine,
Journal Citation Reports/Science Edition, EMBase, Scopus and the Elsevier Bibliographic databases. The manuscript management system is completely online and includes a very quick and fair peer-review system, which is all easy to use. Visit http://www.dovepress.com/ testimonials.php to read real quotes from published authors.

Submit your manuscript here: http://www.dovepress.com/international-journal-of-nanomedicine-journal 